\title{
Probing noncommutativities of phase space by using persistent charged current and its asymmetry
}

\author{
Kai Ma, ${ }^{*}$ Ya-Jie Ren, and Ya-Hui Wang \\ Department of Physics, Shaanxi University of Technology, Hanzhong 723001, People's Republic of China
}

(Received 21 July 2017; published 8 June 2018)

\begin{abstract}
Nontrivial algebra structures of the coordinate and momentum operators are potentially important for describing possible new physics. The persistent charged current in a metal ring is expected to be sensitive to the nontrivial dynamics due to noncommutativities of phase space. In this paper, we propose a new asymmetric observable for probing the noncommutativity of momentum operators. We also analyzed the temperature dependence of this observable, and we find that the asymmetry holds at a finite temperature. The critical temperature, above which the correction due to coordinate noncommutativity is negligible, is also derived.
\end{abstract}

DOI: 10.1103/PhysRevD.97.115011

\section{INTRODUCTION}

One of the most important ingredients of quantum mechanics is the noncommutativity of physical quantities. Evolution of the quantum system is governed by the elementary noncommutative algebras. The commutators among coordinate and momentum operators, $\left[x_{i}, x_{j}\right]=0$, $\left[p_{i}, p_{j}\right]=0,\left[x_{i}, p_{j}\right]=i \hbar \delta_{i j}$, are the fundamental algebras and go into all quantum phenomena. However, there is no any guarantee that can protect the above commutators from possible deviations. The noncommutative space (we will use the notation $X^{\mu}$ to denote the coordinate vectors in this space), which is characterized by the algebra

$$
\left[X_{\mu}, X_{\nu}\right]=i \theta_{\mu \nu}
$$

is an extension of those commutation relations and parametrized by the totally antisymmetric constant tensor $\theta_{\mu \nu}$, which has the dimension of length squared. Mathematically, the noncommutative space can be a candidate of the physical background space-time. On the other hand, noncommutative space can be an effective description of some new physics models at low energy, for instance, string theory embedded in a background magnetic field [1] and quantum gravity [2-5]. Nevertheless, noncommutative property can also appear in a real quantum system [6,7]. Because the noncommutative extension is fundamental, all the ordinary quantum physics are deformed with corrections parametrized by the constant

makainca@yeah.net

Published by the American Physical Society under the terms of the Creative Commons Attribution 4.0 International license. Further distribution of this work must maintain attribution to the author(s) and the published article's title, journal citation, and DOI. Funded by SCOAP. $\theta_{\mu \nu}$, for instance, the breaking of rotational symmetry [8,9], distortion of energy levels of the atoms [10-12], corrections on the spin-orbital interactions [13-20], and the quantum speed of relativistic charged particles [21-25] and fluid dynamics [26,27].

Most importantly, the spatial component of the noncommutative parameter $\left(\theta^{\mu \nu}\right), \theta^{i j}$, behaves like a permanent magnetic dipole moment and gives a contribution $\epsilon_{i j k} \theta^{i j} B^{k}$ to the energy of system. This correction can affect the electromagnetic dynamics of charged particles. It has been shown in Refs. [28-38] that magnetic flux due to the permanent dipole moment can modify the Aharonov-Bohm effect [39] as well as the Aharonov-Casher effect [40]. Furthermore, the magnetic flux can also generate a persistent charged current in a metal ring. In Ref. [41], it was shown that the persistent charged current [42-44] is sensitive to the spatial noncommtuativity and can be employed to probe the low-energy residual effects of possible new physics at the high-energy scale. However, the analysis in Ref. [41] was done by using a simple shift method that in general cannot lead to a gauge-invariant result $[31,32,38,45]$. Furthermore, the persistent current in the real metal ring is also affected by temperature, cross section, etc. Therefore, a more realistic investigation is necessary. In this paper, we will use the Seiberg-Witten (SW) map [1], which preserves the gauge symmetry to study the noncommutative effects on the persistent current in a metal ring, and then analyze temperature dependence of the noncommutative corrections.

The contents of this paper are organized as follows, In Sec. II, we introduce the Hamiltonian of a charged particle on noncommutative phase space. In Sec. III, we will study noncommutative corrections on the persistent current in a metal ring and introduce a new asymmetry observable for probing the noncommutativity of phase space. In Sec. IV, 
we study the temperature dependence of the persistent current as well as thermal effects on the asymmetry observable proposed in Sec. III. Our conclusions are given in the final section, Sec. V.

\section{NONCOMMUTATIVE HAMILTONIAN}

The noncommutative extension of the underlying spacetime is characterized by the algebra in (1). However, in consideration that the momentum operators are defined as the derivatives with respect to coordinates, it is natural to consider a further extension with noncommutative momentum operators defined by the algebra (we will use the notation $P^{\mu}$ to denote the momentum vectors in this space) $[35,41,45-51]$,

$$
\left[P_{\mu}, P_{\nu}\right]=i \xi_{\mu \nu}
$$

where $\xi_{\mu \nu}$ is also a totally antisymmetric constant tensor with the dimension of energy squared. Physical effects of the above extension have been studied in various aspects [35,41,45-51]. And recently, a more general extension on the generators of the whole Poincaré group was conducted in Ref. [52]. Therefore, there are strong phenomenological motivations to study the extensions (1) and (2) simultaneously.

The two parameters, $\theta_{\mu \nu}$ and $\xi_{\mu \nu}$, parametrize the electromagnetic interactions on noncommutative space and scale any new physics due to nontriviality of the background space-time. In general, all their components can be nonzero. However, nonvanishing terms $\theta^{0 i}$ and/or $\xi^{0 j}$ can violate unitarity; therefore, in this paper, we consider noncommutativities of only the spatial components of coordinates and momenta. While the coordinate noncommutativity cannot be taken into account by using a simple shift method, the representation in Eq. (3) in terms of ordinary coordinate and momentum operators can realize the algebra in (2) [45],

$$
P_{\mu}=p_{\mu}-\frac{1}{2 \hbar} \xi_{\mu \nu} x^{\nu},
$$

and can give gauge-invariant physical contributions $[45,50]$. According to this representation, the Lagrangian for a charged particle interacting with external electromagnetic fields can be written as [50]

$$
\mathcal{L}=\bar{\psi}(x)\left(\not p-Q A_{N C}-m\right) \psi(x),
$$

where $Q$ is the electric charge of the particle in units of $|e|$ and the total potential $A_{N C}^{\mu}$ is a sum of the original one $A^{\mu}$ and an effective term $A_{\xi}^{\mu}$ emerging from the transformation (3),

$$
A_{N C}^{\mu}=A^{\mu}+A_{\xi}^{\mu}, \quad A_{\xi}^{\mu}=\frac{\xi}{2 \hbar Q}(0,-y, x, 0) .
$$

In the above derivation of the explicit expression of $A_{\xi}^{\mu}$, we have defined the $\hat{z}$ axis along the direction of the vector $\vec{\xi}$, where the components of $\vec{\xi}$ are related to $\xi^{i j}$ by the relation $\xi_{k}=\epsilon_{i j k} \xi^{i j} / 2$. In this configuration, the nonzero components are only $\xi^{12}=-\xi^{21}=\xi$, and the effect of momentum noncommutativity is an addition of a constant magnetic background field,

$$
\vec{B}_{\xi}=\vec{\nabla} \times \vec{A}_{\xi}=\left(0,0, B_{\xi}\right), \quad B_{\xi}=\frac{\xi}{\hbar Q},
$$

over the whole space.

For the coordinate noncommutativity, it has been shown that the SW map [1] from the noncommmutative space to the ordinary one preserves the gauge symmetry and has been proven to be very useful in investigating various problems on noncommutative space [38,53-60]. Therefore, we will use the SW map to study the noncommutative corrections induced by the nontrivial algebra in (1). For the $U(1)$ gauge symmetry, the SW map is given by

$$
\begin{gathered}
\psi \rightarrow \psi-\frac{1}{2} Q \theta_{\alpha \beta} A_{N C}^{\alpha} \partial^{\beta} \psi, \\
A_{N C}^{\mu} \rightarrow A_{N C}^{\mu}-\frac{1}{2} Q \theta_{\alpha \beta} A_{N C}^{\alpha}\left(\partial^{\beta} A_{N C}^{\mu}+F_{N C}^{\beta \mu}\right),
\end{gathered}
$$

where $F_{N C}^{\mu \nu}=\partial^{\mu} A_{N C}^{\nu}-\partial^{\nu} A_{N C}^{\mu}$ is the effective electromagnetic field strength tensor. Then, in terms of the ordinary fields, the noncommutative Lagrangian (4) can be written as

$$
\begin{aligned}
\mathcal{L}_{N C}= & \left(1-\frac{1}{4} Q \theta_{\alpha \beta} F_{N C}^{\alpha \beta}\right) \bar{\psi}(x)\left(i \gamma_{\mu} D_{N C}^{\mu}-m\right) \psi(x) \\
& -\frac{i}{2} Q \theta_{\alpha \beta} \bar{\psi}(x) \gamma^{\mu} F_{N C}^{\mu \alpha} D_{N C}^{\beta} \psi(x),
\end{aligned}
$$

where $D_{N C}^{\beta}=\partial^{\beta}+i Q A_{N C}^{\beta}$ is the covariant derivative. This Lagrangian is gauge invariant because the noncommutative corrections depend only on the covariant derivative $D_{N C}^{\beta}$ and the electromagnetic field strength $F_{N C}^{\mu \nu}$. Therefore, the noncommutative corrections on the Aharonov-Bohm phase shift can be defined unambiguously [28-33] and can be interpreted consistently on the commutative and noncommutative phase spaces. The equation of motion can be obtained directly from this Lagrangian as

$$
\begin{aligned}
& \left(i \gamma_{\mu} D_{N C}^{\mu}-m\right) \psi(x)-\frac{i}{2} Q\left(1-\frac{1}{4} Q \theta_{\alpha \beta} F_{N C}^{\alpha \beta}\right)^{-1} \\
& \times \theta_{\alpha \beta} \gamma^{\mu} F_{N C}^{\mu \alpha} D_{N C}^{\beta} \psi(x)=0,
\end{aligned}
$$

where we have multiplied a factor $\left(1-Q \theta_{\alpha \beta} F_{N C}^{\alpha \beta} / 4\right)^{-1}$ to simplify the expression. By expanding $\left(1-Q \theta_{\alpha \beta} F_{N C}^{\alpha \beta} / 4\right)^{-1}$ 
with respect to the noncommutative parameter $\theta^{\mu \nu}$, one can see that the correction proportional to $Q \theta_{\alpha \beta} F_{N C}^{\alpha \beta}$ can be neglected at the first order of the noncommutative parameter $\theta^{\mu \nu}$. In some studies, this term was taken into account through the renormalization of the particle charge. However, for consistency, from here on, we will keep only the leading-order terms. Therefore, we will consider only the correction that involves the covariant derivative. Under this approximation, the equation of motion can be written as

$$
\begin{gathered}
\left(i \gamma_{\mu} \mathcal{D}_{N C}^{\mu}-m\right) \psi(x)=0 \\
\mathcal{D}_{N C}^{\mu}=\left(\eta^{\mu}{ }_{\beta}-\frac{1}{2} Q F_{N C}^{\mu \alpha} \theta_{\alpha \beta}\right) D_{N C}^{\beta} \equiv g^{\alpha}{ }_{\beta} D_{N C}^{\beta} .
\end{gathered}
$$

The deformed Dirac equation is similar to the Dirac equation in curved space-time with the metric $g_{\alpha \beta}$. The leading-order effect of the noncommutative phase space behaves like a perturbation on the flat space-time defined by the metric $\eta_{\alpha \beta}$. The perturbation depends on the external magnetic field, noncommutative parameters, and their relative angles. The corresponding Dirac Hamiltonian resulting in the equation of motion (11) is

$$
H_{N C}=\beta m+g_{i j} \alpha^{i}\left(p^{j}-Q A_{N C}^{j}\right)+Q g_{00} V_{N C} .
$$

The nonrelativistic approximation can be obtained by using the well-known Foldy-Wouthuysen (FW) unitary transformation [61], which approximately block diagonalizes the Dirac Hamiltonian by separating the positive and negative energy parts of its spectrum. Neglecting the spin degree of freedom, the nonrelativistic Hamiltonian is

$$
H_{N C}=\frac{1}{2 m_{\theta}}\left(\vec{p}-Q \vec{A}_{N C}\right)^{2},
$$

where

$$
m_{\theta}=\frac{m}{1-Q \frac{\vec{\theta} \cdot \vec{B}_{N C}}{2 \phi_{0}}}
$$

is the effective mass on the noncommutative phase space and $\phi_{0}=h / e$ is the fundamental magnetic flux. Because $|\theta||\xi| \ll \hbar^{2}$, and furthermore usually the external magnetic field $|\vec{B}|$ is much stronger then the noncommutative background $\left|\vec{B}_{\xi}\right|$, the mass renormalization effect given in (15) can be approximated as $m_{\theta} \approx m\left[1-Q \vec{\theta} \cdot \vec{B} /\left(2 \phi_{0}\right)\right]^{-1}$. This means that, in the nonrelativistic limit, coordinate noncommutativity only affects the effective mass of charged particles.

\section{PERSISTENT CURRENT AND ASYMMETRY}

The noncommutativity of space-time can distort the electromagnetic dynamical behavior of charged carriers, and in the nonrelativistic limit, their properties are essentially determined by the Hamiltonian given in (14). In this section, we study the noncommutative effects on the persistent charged current in a metal ring [41-44]. We will ignore the effects due to the finite cross section of the metal ring, such that motion of the electrons is confined in a ring with radius $R$. We choose a reference frame in which the ring lies in the $x y$ plane and is centered on the origin, and the dynamical variable is only the azimuthal angle variable $\varphi$ in this plane. In the presence of a universal external magnetic field $\vec{B}=(0,0, B)$, according to the Hamiltonian given in (14), the corresponding static noncommutative Schrödinger equation is

$$
\frac{1}{2 m_{\theta}}\left(-i \hbar \frac{1}{R} \frac{d \psi}{d \varphi}+\frac{e B_{N C} R}{2}\right)^{2} \psi_{n}=\epsilon_{n} \psi_{n},
$$

where $B_{N C}=B-B_{\xi}$ is the noncommutative corrected magnetic field and the symmetric gauge was used in the above derivation. Furthermore, we have set $Q=-e$ for the electron. The solutions of (16) are $\psi_{n}=e^{i n \varphi} / \sqrt{2 \pi}$ with integral quantum numbers $n$ and energy levels

$$
\epsilon_{n}=\frac{h^{2}}{2 m_{\theta} L^{2}}\left(n+\frac{\phi_{N C}}{\phi_{0}}\right)^{2},
$$

where $\phi_{N C}=\pi R^{2} B_{N C}$ is the total magnetic flux and $L=2 \pi R$ is the length of the metal ring. Similar to the results in ordinary phase space, for a given quantum number $n$, the energy eigenvalues $\epsilon_{n}$ are a parabola in the magnetic flux $\phi_{N C}$. Furthermore, the energy levels are degenerate for $n=N$ with $-1 / 2<\phi_{N C} / \phi_{0}<1 / 2$ and $n=N+l$ with $l-1 / 2<\phi_{N C} / \phi_{0}<l+1 / 2$, where $l$ is an arbitrary integer. This property results in periodic velocity and hence a periodic charged current. By using the Heisenberg equation, one can easily obtain the expectation value of the one-particle mechanical velocity operator at the quantum state $\psi_{n}$,

$$
v_{n}=\frac{h}{m_{\theta} L}\left(n+\frac{\phi_{N C}}{\phi_{0}}\right) .
$$

The corresponding charged current can be obtained by multiplying $v_{n}$ by $-e / L$ since the electron turns through a given point $v_{n} / L$ times in a unite time,

$$
i_{n}=-\frac{e h}{m_{\theta} L^{2}}\left(n+\frac{\phi_{N C}}{\phi_{0}}\right) .
$$

While persistent currents are usually associated with superconductors, it can also exist in some resistive material as well, as long as the mean scattering length is considerably larger than the length of ring [42-44]. The current (19) is the single-level contribution to the persistent charged current. In case of a real ring, currents from many electrons have to be summed up. In consideration of the fact that the long-range interactions within the metal are suppressed due to the charge screening effect, we will 
neglect the interactions among those electrons. This approximation is not straightforward because the noncommutative correction is expected to be small. Therefore, this approximation is valid only in the case in which the noncommutative correction is larger than the contributions of long-range interactions. Here, we simply assume this approximation is justified and discuss it when the temperature effect is included.

For $0<\phi_{N C}<\phi_{0} / 2$, the energy levels are arranged in ascending order, i.e., $0,-1,+1,-2,+2, \ldots$. For $-\phi_{0} / 2<$ $\phi_{N C}<0$, the ordering of levels is reversed for each $n$. Because of the linearity of the current in quantum number $n$, the contributions from positive integer $+n$ and negative integer $-n$ cancel each other. Therefore, in case the levels are filled by both positive and negative quantum numbers $n$, the net contribution is linearly proportional to the magnetic flux $\phi_{N C}$. Denoting the highest level filled with both positive and negative integers as $N$, then the total current in this case is

$$
I_{N}=-\frac{e h}{m_{\theta} L^{2}}(4 N+2) \frac{\phi_{N C}}{\phi_{0}} .
$$

Here, we have taken into account of the twofold degeneracy of each level due to spin. This result is also valid for $-\phi_{0} / 2<\phi_{N C}<\phi_{0} / 2$ since the cancellation explained above happens in spite of the sign of the magnetic flux $\phi_{N C}$. The contributions of the next four electrons to the total current are

$$
\begin{gathered}
I_{N+1}^{1}=I_{N}-\frac{e h}{m_{\theta} L^{2}}\left(-\sigma(N+1)+\frac{\phi_{N C}}{\phi_{0}}\right), \\
I_{N+1}^{2}=I_{N}-\frac{2 e h}{m_{\theta} L^{2}}\left(-\sigma(N+1)+\frac{\phi_{N C}}{\phi_{0}}\right), \\
I_{N+1}^{3}=I_{N}-\frac{e h}{m_{\theta} L^{2}}\left(-\sigma(N+1)+3 \frac{\phi_{N C}}{\phi_{0}}\right), \\
I_{N+1}^{4}=I_{N}-\frac{4 e h}{m_{\theta} L^{2}} \frac{\phi_{N C}}{\phi_{0}},
\end{gathered}
$$

where $\sigma=\operatorname{sgn}\left(\phi_{N C}\right)$ is the sign of $\phi_{N C}$ and the superscript $I_{N+1}$ is used to indicate the number of electrons that have been added to the system. One can clearly see that, due to the cancellation, the total current always has a magnitude of the order of the current contribution from the highest energy. At a static state with $n=N$, according to Eq. (18), the Fermi velocity of the $N$ th level at $\phi_{N C}=0$ is $v_{F}=h N / m_{\theta} L$. Therefore, no matter how the energy levels are filled, the typical magnitude of the total current is of order $I_{0}=e v_{F} / L$. Since this value has the same magnitude as that of the electron in the highest occupied level, each additional electron has a strong effect on the flux dependence of the current. Therefore, the magnetic flux dependence is sensitive to the number of electrons in the ring. In Ref. [41], the authors studied the currents by distinguishing even and odd numbers of electrons. However, for an ensemble of rings, the number of electrons has a spread, and hence averaging the currents among different number of electrons is necessary. Therefore, we will not study the currents separately; we are aiming to find observables that are independent of the number of electrons.

From above results, one can see that the charged current receives noncommutative corrections in two ways. The first one is through the renormalization of the electron mass, and hence of the Fermi velocity $v_{F}=h N / m_{\theta} L$. This correction is independent of the number of electrons (in the limit $N \gg 1$ ). However, the noncommutative correction is too small; for $\sqrt{\theta} \sim 1 \mathrm{fm}$, the correction is at the order of $10^{-15}$. Therefore, for an ideal ring, the correction due to the noncommutativity of coordinate operators is completely negligible. In the case of finite temperature, the Fermi velocity is affected by thermal fluctuation, and hence there is hope for probing the $\theta$-dependent correction through the thermal behavior. However, we will shown in next section that this is also challengeable.

The second one is the violation of the symmetry property of the persistent current. In the ordinary phase space, i.e., $\phi_{N C}=\phi=\pi R^{2} B$, the current is antisymmetric about $\phi_{N C}=0$, i.e., $I\left(-\phi_{N C}\right)=-I\left(\phi_{N C}\right)$, and hence the null point is defined by the condition $B=0$, i.e., $I(-B)=$ $-I(B)$. Nevertheless, on noncommutative phase space, this null point is shifted by the noncommutative correction, which is independent of the external magnetic field $\vec{B}$, i.e., $I(-B)=-I(B)+2 I_{\xi}$, where $I_{\xi}$ denotes the $B$-independent part of the current $I(B)$. Therefore, an asymmetric observable can be defined to probe the noncommutativity of the momentum space. Here, we introduce the asymmetry observable to measure the noncommutative effects,

$$
\mathcal{A}_{\xi}=\frac{I(B)+I(-B)}{I(B)-I(-B)}=\frac{I_{\xi}}{I_{B}},
$$

where $I_{B}$ denotes the $B$-dependent part of the current $I(B)$. The advantage of this observable is that it is a function of only external magnetic field and hence is stable against the interaction between electrons. For an idea ring, the persistent current is given by (20); thus, the asymmetry can be written as

$$
\mathcal{A}_{\xi}=-\frac{1}{e \hbar} \frac{\xi}{B} .
$$

Clearly, this asymmetry vanishes in the limit $\xi \rightarrow 0$. On the other hand, the asymmetry $\mathcal{A}_{\xi}$ is independent of the noncommutative parameter $\theta$, and hence it measures only the noncommutativity of the momentum space. Furthermore, the sign of the noncommutative parameter $\xi$ can also be measured. This asymmetry is also affected by thermal fluctuation, and the details are given in the next section. 


\section{TEMPERATURE DEPENDENCE}

In last section, we obtained the noncommutative corrections on the persistent charged current generated by the external magnetic field in a metal ring. In this section, we study their temperature dependence. For convenience, we will use another expression of the single-level current to study the thermal effects. In the last section, we derive the charged current by using the Heisenberg equation. In fact, it can also be obtained by differentiating the energy eigenvalues with respect to external magnetic flux,

$$
i_{n}=-\frac{\partial \epsilon_{n}}{\partial \phi}
$$

We have checked that these two approaches lead to the same results in our case. In this approach, the total current at temperature $T$ can be written as

$$
I=\sum\left(-\frac{\partial \epsilon_{n}}{\partial \phi}\right) f\left(\epsilon_{n}, \mu, T\right)=-\frac{\partial \Omega}{\partial \phi},
$$

where $f\left(\epsilon_{n}, \mu, T\right)$ is the Fermi-Dirac distribution function and $\Omega$ is the grand potential of thermodynamics. In our case, the grand canonical potential can be written as

$$
\Omega=-\frac{1}{\beta} \int_{-\infty}^{\infty} d \epsilon \varrho\left(\epsilon, \phi_{N C}\right) \ln \left(1+e^{-\beta\left(\epsilon-\epsilon_{F}\right)}\right),
$$

where $\beta^{-1}=k_{B} T ; \epsilon_{F}$ is the Fermi energy, and $\varrho\left(\epsilon, \phi_{N C}\right)=$ $\sum_{n} g_{n} \delta\left(\epsilon-\epsilon_{n}\left(\phi_{N C}\right)\right)$ is the flux-dependent density of states with degeneracy $g_{n}$. In the following analysis, we will neglect the spin-dependent interactions, and in this approximation, $g_{n}=2$. Rewriting the density of states in terms of its harmonics, and performing integration by parts, one can obtain

$$
\begin{aligned}
\Omega= & \int_{-\infty}^{\infty} d \epsilon\left\{\sum_{p>0} \frac{4 \hbar^{2} k \cos \left(2 \pi p \nu_{N C}\right)}{m_{\theta} \pi p^{2} L}\right. \\
& \times\left(\frac{\sin (p k L)}{p k L}-\cos (p k L)\right) \\
& \left.+\frac{8}{3} \sqrt{\frac{2 m_{\theta} L^{2}}{h^{2}}} \epsilon^{3 / 2}\right\} f^{\prime}\left(\epsilon, \epsilon_{F}, T\right),
\end{aligned}
$$

where $\nu_{N C}=\phi_{N C} / \phi_{0}$ and the function $f^{\prime}\left(\epsilon, \epsilon_{F}, T\right)$ is the derivative of Fermi-Dirac distribution and given by the expression

$$
f^{\prime}\left(\epsilon, \epsilon_{F}, T\right)=\frac{1}{4} \beta \sinh ^{2}\left[\frac{1}{2} \beta\left(\epsilon-\epsilon_{F}\right)\right],
$$

which is peaked around the Fermi energy $\epsilon_{F}$ with characteristic width $\beta^{-1}$. The corresponding current can be obtained by using (28),

$$
\begin{aligned}
I= & -\int_{-\infty}^{\infty} d \epsilon \sum_{p>0} \frac{8 \hbar^{2} k \sin \left(2 \pi p \nu_{N C}\right)}{m_{\theta} p L \phi_{0}} \\
& \times\left(\frac{\sin (p k L)}{p k L}-\cos (p k L)\right) f^{\prime}\left(\epsilon, \epsilon_{F}, T\right) .
\end{aligned}
$$

Typically, the temperature $k_{B} T$ of the metal system is much less than the Fermi energy $\epsilon_{F}$, i.e., $k_{B} T \ll \epsilon_{F}$; therefore, the factor $k$ will be of order $k_{F}=\sqrt{2 m_{\theta} \epsilon_{F}} / \hbar$ in the integrand. For typical Fermi velocity $v_{F} \approx 10^{6} \mathrm{~m} / \mathrm{s}$, $k_{F} \approx 8.7 \times 10^{9} \mathrm{~m}^{-1}$, the product $k_{F} L=8.7 \times 10^{3}$ is much larger than 1 for a typical length of ring $L=1 \mathrm{~mm}$. Therefore, contributions from the oscillation term $\sin (p k L) /(p k L)$ in the integral (32) can be safely neglected. The contributions from the term proportional to $\cos (p k L)$ can be calculated perturbatively by expanding $k$ around $k_{F}$,

$$
k \approx k_{F}\left(1+0.5 r+\mathcal{O}\left(r^{2}\right)\right),
$$

where $r=\epsilon / \epsilon_{F}-1$ is a small quantity as explained above. In the following calculations, we will keep only the terms linear in $r$. This approximation simplifies the calculations; however, some conditions must be satisfied in order to get reasonable noncommutative corrections. The leading-order noncommutative correction on $k$ is given by

$$
\hbar \delta_{N C} k=\frac{\theta B}{\phi_{0}} m v_{F},
$$

and the second-order thermal contribution is given by

$$
\hbar \delta_{\mathrm{th}} k=\frac{1}{8}\left(\frac{k_{B} T}{\epsilon_{F}}\right)^{2} m v_{F} .
$$

Therefore, the critical temperature, above which the $\theta$-dependent noncommutative correction will be washed out by the thermal motion, is given as follows:

$$
T_{\theta}=\frac{2 m v_{F}^{2}}{k_{B}} \sqrt{\frac{\theta B}{\phi_{0}}} .
$$

In Fig. 1, we show the dependence of the critical temperature $T_{\theta}$ on noncommutative parameter $\theta$. The black solid line shows $T_{\theta}$ for external magnetic field $B=1 \mathrm{~T}$, while the red-dashed line shows the case of $B=10 \mathrm{~T}$. Since the Fermi velocity $v_{F}$ is relatively stable among most of the metals, we have used a typical value $v_{F}=1 \times 10^{6} \mathrm{~m} \cdot \mathrm{s}^{-1}$ in the estimation. We can see from the figure that for a space noncommutativity at the $\mathrm{TeV}$ scale a temperature below $1 \mathrm{mK}$ is necessary, which is challengeable for now. Therefore, in the following discussions, we will neglect this correction. 


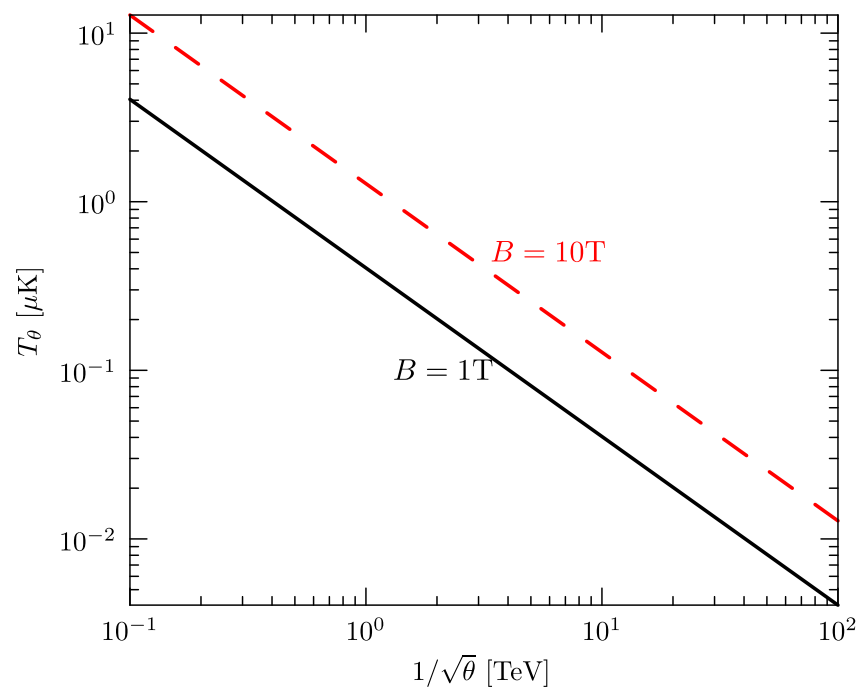

FIG. 1. Critical temperature $T_{\theta}$ for observing the noncommutative correction on the total current. The black solid line shows $T_{\theta}$ for the external magnetic field $B=1 \mathrm{~T}$, while the red dashed line shows the case of $B=10 \mathrm{~T}$. In addition, a typical Fermi velocity $v_{F}=1 \times 10^{6} \mathrm{~m} \cdot \mathrm{s}^{-1}$ is used in both cases.

In this approximation, the temperature-dependent current is given as

$$
I=\frac{4}{\pi} I_{0} \sum_{p>0} p^{-1} \sin \left(2 \pi p \nu_{N C}\right) \cos \left(p k_{F} L\right) g\left(\kappa_{p}\right),
$$

where the factor $\kappa_{p}=T / T_{p}$ with a typical temperature $T_{p}=\hbar v_{F} /\left(2 \pi p k_{B} L\right)$ and the function $g\left(\kappa_{p}\right)$ is defined as

$$
g\left(\kappa_{p}\right)=\frac{\kappa_{p}}{\sinh \left(\kappa_{p}\right)} .
$$

For $T>T_{p}$, the $p$ th harmonic of the current decays exponentially as the temperature increases. Furthermore, the total current is sinusoidal in the magnetic flux, which is different from the linearity as given in (20). Figure 2 shows such oscillations in cases of different temperature and noncommutative parameters $\xi$. The black solid line shows the total current at $T=1 \mathrm{~K}$ on ordinary phase space. As expected, it pass through the null point. The red dashed line shows the total current at the same temperature but $\sqrt{\xi}=1 \mathrm{eV}$. One can see clearly that the null point has been shifted. The blue dotted line shows the total current with $\sqrt{\xi}=1 \mathrm{eV}, T=1 \mathrm{mK}$. As expected, the magnitude of the total current is enhanced by lowering the temperature. The purple dashed-dotted line shows the case of $\sqrt{\xi}=1 \mathrm{meV}$,

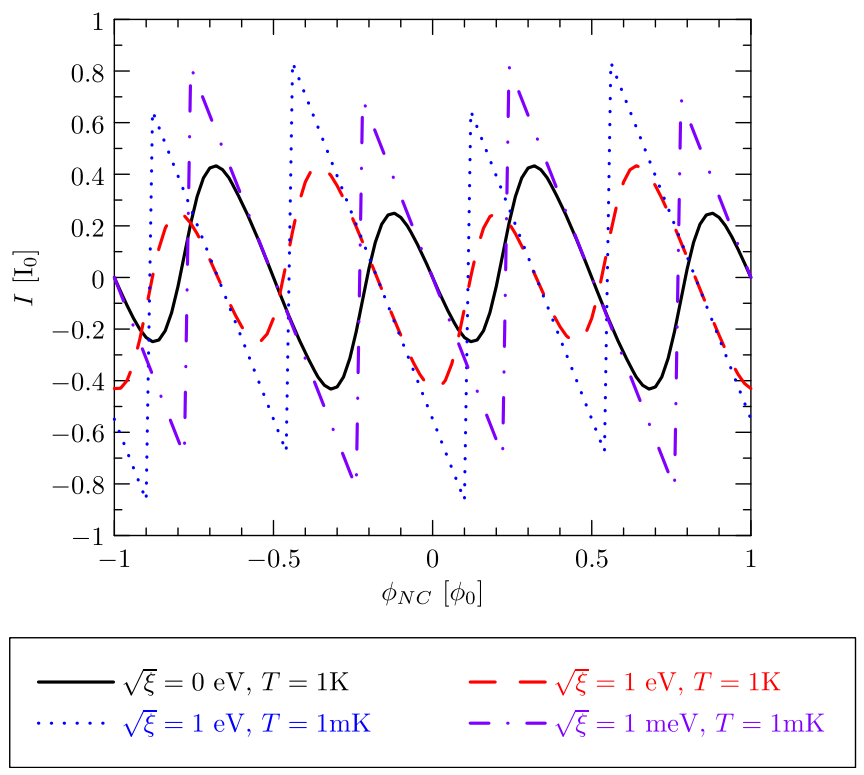

FIG. 2. Oscillation and the shift of the null-point of the total current in case of finite temperature. The total current is given in unite of $I_{0}$ and the magnetic flux $\phi_{N C}$ is given in unite of $\phi_{0}$. The black-solid line shows the total current for $\xi=0$ and $T=1 \mathrm{~K}$. The red-dashed line shows the case for $\sqrt{\xi}=1 \mathrm{~K}$ and $T=1 \mathrm{~K}$. The blue-dotted line shows $I$ for $\sqrt{\xi}=1 \mathrm{eV}, T=0.1 \mathrm{mK}$, and purple-dash-dotted line is for the case of $\sqrt{\xi}=1 \mathrm{meV}$, $T=1 \mathrm{mK}$. In all cases, $v_{F}=1 \times 10^{6} \mathrm{~m} \cdot \mathrm{s}^{-1}$ and $L=1 \mu \mathrm{m}$ are used.

$T=1 \mathrm{mK}$. One can see that an approximately linear dependence is recovered, and the null point closes to the origin.

Furthermore, according to the definition of the asymmetry given in (25), the temperature-dependent asymmetry is

$\mathcal{A}_{\xi}=-\frac{\sum_{p>0} p^{-1} \sin \left(2 \pi p \nu_{\xi}\right) \cos (2 \pi p \nu) \cos \left(p k_{F} L\right) g\left(\kappa_{p}\right)}{\sum_{p>0} p^{-1} \cos \left(2 \pi p \nu_{\xi}\right) \sin (2 \pi p \nu) \cos \left(p k_{F} L\right) g\left(\kappa_{p}\right)}$,

where $\nu=\phi / \phi_{0}$ and $\nu_{\xi}=\phi_{\xi} / \phi_{0}$. However, since denominator of $\mathcal{A}_{\xi}$ is sinusoidal in the filling factor $\nu$, occasionally the value of $\mathcal{A}_{\xi}$ can be unreasonably huge. Therefore, it is more convenient to define an averaged asymmetry over a certain range of the external magnetic flux. In consideration of the fact that the terms with $p=1$ have relatively larger contributions, we chose the range $0 \leq \nu \leq 1 / 4$ as a benchmark point, and the corresponding averaged asymmetry is given as

$$
\overline{\mathcal{A}}_{\xi}=-\frac{\int_{0}^{1 / 4} d \nu \sum_{p>0} p^{-1} \sin \left(2 \pi p \nu_{\xi}\right) \cos (2 \pi p \nu) \cos \left(p k_{F} L\right) g\left(\kappa_{p}\right)}{\int_{0}^{1 / 4} d \nu \sum_{p>0} p^{-1} \cos \left(2 \pi p \nu_{\xi}\right) \sin (2 \pi p \nu) \cos \left(p k_{F} L\right) g\left(\kappa_{p}\right)} .
$$




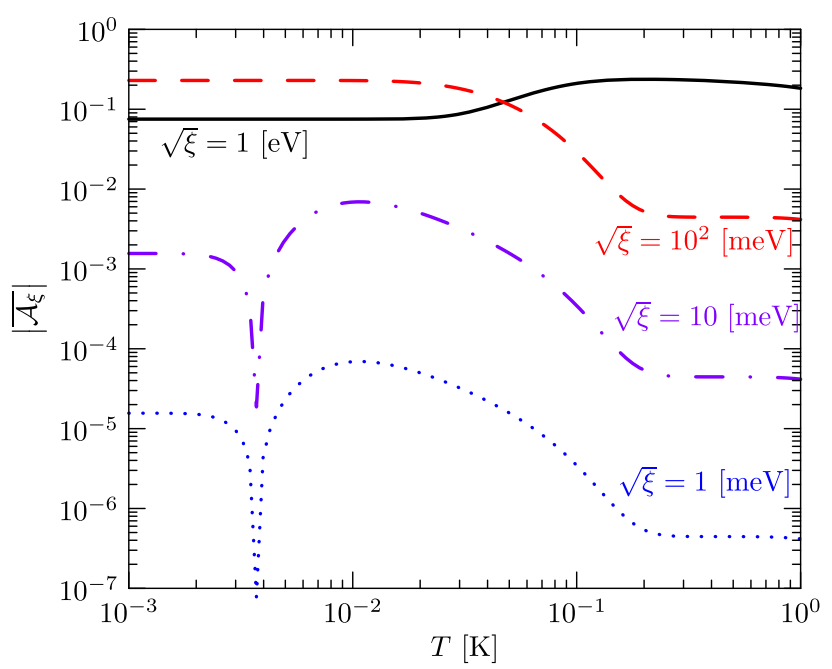

FIG. 3. Temperature dependence of the magnitude of the averaged asymmetry. The black solid, red dashed, purple dashed-dotted, and blue dotted lines show the cases of $\sqrt{\xi}=$ $1 \mathrm{eV}, \sqrt{\xi}=10^{2} \mathrm{meV}, \sqrt{\xi}=10 \mathrm{meV}$, and $\sqrt{\xi}=1 \mathrm{meV}$, respectively. In all cases, $v_{F}=1 \times 10^{6} \mathrm{~m} \cdot \mathrm{s}^{-1}$, and $L=1 \mu \mathrm{m}$ are used.

Figure 3 shows the temperature dependence of the magnitude of the averaged asymmetry for different values of the noncommutative parameter $\xi$. Oscillation of the asymmetry is due to the relative importance of the contribution of the $p$ th harmonic being affected by the temperature. From the figure, one can also see that for $\sqrt{\xi} \sim \mathrm{meV}$ the asymmetry $\overline{\mathcal{A}}_{\xi}$ is at an order of $10^{-4}$.

\section{CONCLUSIONS}

In summary, we have studied the noncommutative corrections on the persistent charged current in a metal ring as well as their temperature dependence. Based on the
SW map, the nonrelativistic Hamiltonian on a noncommutative phase space was obtained by using the FW transformation. At the leading order of noncommutative parameters, the corrections due to coordinate and momentum noncommutativity come into play through the mass renormalization and shift of the external magnetic flux, respectively. Because the mass renormalization affects only the total magnitude of the current, it is challenging to measure the coordinate noncommutative parameter $\theta$. However, the noncommutativity of momentum can violate the symmetry property of the persistent current. Furthermore, this violation effect still exists at a finite temperature. Based on this violation effect, we have introduced an asymmetry observable, which is sensitive to the momentum noncommutativity, to measure the corresponding parameter $\xi$. In practice, the finite cross section of the metal ring also affects the persistent current. However, the symmetry property is preserved even in this case. Therefore, the observable introduced in our paper is essential to probe the momentum noncommutativity. It has been reported that the upper bound of the noncommutative parameter $\xi$ is $\sqrt{\xi}<1 \mathrm{meV}$ for $C P T$-violating noncommutative interaction [51]. A similar bound was also reported by investigating the noncommutative corrections in a quantum gravitational well [62]. However, those bounds are model dependent and hence cannot be used to rule out the possibility of observing the noncommutativity of momentum operators by using the persistent charged current.

\section{ACKNOWLEDGMENTS}

K. M. and Y. H. W. are supported by the National Natural Science Foundation of China under Grant No. 11705113 and partially by the Shaanxi Natural Science Foundation Project under Grant No. 2017JM1032.
[1] N. Seiberg and E. Witten, String theory and noncommutative geometry, J. High Energy Phys. 09 (1999) 032.

[2] L. Freidel and E. R. Livine, Effective 3-D Quantum Gravity and Non-Commutative Quantum Field Theory, Phys. Rev. Lett. 96, 221301 (2006).

[3] J. W. Moffat, Noncommutative quantum gravity, Phys. Lett. B 491, 345 (2000).

[4] J. W. Moffat, Perturbative noncommutative quantum gravity, Phys. Lett. B 493, 142 (2000).

[5] M. Faizal, Noncommutative quantum gravity, Mod. Phys. Lett. A 28, 1350034 (2013).

[6] J. Gamboa, M. Loewe, and J. C. Rojas, Noncommutative quantum mechanics, Phys. Rev. D 64, 067901 (2001).
[7] P.-M. Ho and H.-C. Kao, Noncommutative Quantum Mechanics from Noncommutative Quantum Field Theory, Phys. Rev. Lett. 88, 151602 (2002).

[8] M. R. Douglas and N. A. Nekrasov, Noncommutative field theory, Rev. Mod. Phys. 73, 977 (2001).

[9] R. J. Szabo, Quantum field theory on noncommutative spaces, Phys. Rep. 378, 207 (2003).

[10] M. Chaichian, M. M. Sheikh-Jabbari, and A. Tureanu, Hydrogen Atom Spectrum and the Lamb Shift in Noncommutative QED, Phys. Rev. Lett. 86, 2716 (2001).

[11] J.-z. Zhang, Testing Spatial Noncommutativity via Rydberg Atoms, Phys. Rev. Lett. 93, 043002 (2004). 
[12] B.-Q. Wang, Z.-W. Long, C.-Y. Long, and S.-R. Wu, Solution of the spin-one DKP oscillator under an external magnetic field in noncommutative space with minimal length, Chin. Phys. B 27, 010301 (2018).

[13] K. Ma and S. Dulat, Spin Hall effect on a noncommutative space, Phys. Rev. A 84, 012104 (2011).

[14] B. Basu, D. Chowdhury, and S. Ghosh, Inertial spin Hall effect in noncommutative space, Phys. Lett. A 377, 1661 (2013).

[15] J. Wang, K. Ma, K. Li, and H. Fan, Deformations of the spin currents by topological screw dislocation and cosmic dispiration, Ann. Phys. (Amsterdam) 362, 327 (2015).

[16] J.-h. Wang, K. Ma, and K. Li, Influences of a topological defect on the spin Hall effect, Phys. Rev. A 87, 032107 (2013).

[17] A. A. Deriglazov and A. M. Pupasov-Maksimov, Relativistic corrections to the algebra of position variables and spinorbital interaction, Phys. Lett. B 761, 207 (2016).

[18] W. G. Ramrez and A. A. Deriglazov, Relativistic effects due to gravimagnetic moment of a rotating body, Phys. Rev. D 96, 124013 (2017).

[19] Y.-J. Ren and K. Ma, Influences of the coordinate dependent noncommutative space on charged and spin currents, arXiv:1802.10452.

[20] A. A. Deriglazov and W. G. Ramrez, Frame-dragging effect in the field of non rotating body due to unit gravimagnetic moment, Phys. Lett. B 779, 210 (2018).

[21] K. Wang, Y.-F. Zhang, Q. Wang, Z.-W. Long, and J. Jing, Quantum speed limit for a relativistic electron in the noncommutative phase space, Int. J. Mod. Phys. A 32, 1750143 (2017).

[22] K. Wang, Y. F. Zhang, Q. Wang, Z. W. Long, and J. Jing, Quantum speed limit for relativistic spin-0 and spin-1 bosons on commutative and noncommutative planes, Adv. High Energy Phys. 2017, 4739596 (2017).

[23] K. Ma, J.-H. Wang, H.-X. Yang, and H.-W. Fan, Hall conductivity in the cosmic defect and dislocation spacetime, Chin. Phys. Lett. 33, 100401 (2016).

[24] A. A. Deriglazov and W. G. Ramrez, Mathisson-PapapetrouTulczyjew-Dixon (MPTD) equations in ultra-relativistic regime and gravimagnetic moment, Int. J. Mod. Phys. D 26, 1750047 (2017).

[25] A. A. Deriglazov and W. Ramrez, Ultrarelativistic spinning particle and a rotating body in external fields, Adv. High Energy Phys. 2016, 1376016 (2016).

[26] P. Das and S. Ghosh, Noncommutative geometry and fluid dynamics, Eur. Phys. J. C 76, 627 (2016); ErratumEur. Phys. J. C77, 64 (2017).

[27] K. Ma, Noncommutative effects on the fluid dynamics and modifications of the Freidmann equation, arXiv:1801.02533.

[28] M. Chaichian, A. Demichev, P. Presnajder, M. M. SheikhJabbari, and A. Tureanu, Quantum theories on noncommutative spaces with nontrivial topology: Aharonov-Bohm and Casimir effects, Nucl. Phys. B611, 383 (2001).

[29] M. Chaichian, A. Demichev, P. Presnajder, M. M. SheikhJabbari, and A. Tureanu, Aharonov-Bohm effect in noncommutative spaces, Phys. Lett. B 527, 149 (2002).

[30] H. Falomir, J. Gamboa, M. Loewe, F. Mendez, and J. C. Rojas, Testing spatial noncommutativity via the AharonovBohm effect, Phys. Rev. D 66, 045018 (2002).
[31] M. Chaichian, M. Langvik, S. Sasaki, and A. Tureanu, Gauge Covariance of the Aharonov-Bohm Phase in Noncommutative Quantum Mechanics, Phys. Lett. B 666, 199 (2008).

[32] K. Ma, J.-H. Wang, and H.-Yang, Time-dependent AharonovBohm effect on the noncommutative space, Phys. Lett. B 759, 306 (2016).

[33] M. A. Anacleto, F. A. Brito, A. Mohammadi, and E. Passos, Aharonov-Bohm effect for a fermion field in the acoustic black hole "spacetime", Eur. Phys. J. C 77, 239 (2017).

[34] B. Mirza and M. Zarei, Noncommutative quantum mechanics and the Aharonov-Casher effect, Eur. Phys. J. C 32, 583 (2004).

[35] K. Li and J. Wang, The topological AC effect on noncommutative phase space, Eur. Phys. J. C 50, 1007 (2007).

[36] B. Mirza, R. Narimani, and M. Zarei, Aharonov-Casher effect for spin one particles in a noncommutative space, Eur. Phys. J. C 48, 641 (2006).

[37] K. Li, D. Sayipjamal, and J.-H. Wang, The He-McKellarWilkens effect for spin-1 particles on non-commutative space, Chin. Phys. B 17, 1716 (2008).

[38] K. Ma, J.-h. Wang, and H.-X. Yang, Seiberg-Witten map and quantum phase effects for neutral Dirac particle on noncommutative plane, Phys. Lett. B 756, 221 (2016).

[39] Y. Aharonov and D. Bohm, Significance of electromagnetic potentials in the quantum theory, Phys. Rev. 115, 485 (1959).

[40] Y. Aharonov and A. Casher, Topological Quantum Effects for Neutral Particles, Phys. Rev. Lett. 53, 319 (1984).

[41] S.-Dong Liang, H. Li, and G.-Y. Huang, Detecting noncommutative phase space by the Aharonov-Bohm effect, Phys. Rev. A 90, 010102 (2014).

[42] H.-F. Cheung, Y. Gefen, E. K. Riedel, and W.-H. Shih, Persistent currents in small one-dimensional metal rings, Phys. Rev. B 37, 6050 (1988).

[43] A. C. Bleszynski-Jayich, W. E. Shanks, B. Peaudecerf, E. Ginossar, F. von Oppen, L. Glazman, and J. G. E. Harris, Persistent currents in normal metal rings, Science 326, 272 (2009), http://science.sciencemag.org/content/326/5950/272 .full.pdf.

[44] E. Ginossar, L. I. Glazman, T. Ojanen, F. von Oppen, W. E. Shanks, A. C. Bleszynski-Jayich, and J. G. E. Harris, Mesoscopic persistent currents in a strong magnetic field, Phys. Rev. B 81, 155448 (2010).

[45] O. Bertolami and P. Leal, Aspects of phase-space noncommutative quantum mechanics, Phys. Lett. B 750, 6 (2015).

[46] E. Langmann and R. J. Szabo, Duality in scalar field theory on noncommutative phase spaces, Phys. Lett. B 533, 168 (2002).

[47] E. Langmann, R. J. Szabo, and K. Zarembo, Exact solution of quantum field theory on noncommutative phase spaces, J. High Energy Phys. 01 (2004) 017.

[48] K. Li and N. Chamoun, Hydrogen atom spectrum in noncommutative phase space, Chin. Phys. Lett. 23, 1122 (2006).

[49] B. Muthukumar, Remarks on the formulation of quantum mechanics on noncommutative phase spaces, J. High Energy Phys. 01 (2007) 073. 
[50] K. Ma, J.-H. Wang, and H.-X. Yang, Probing the noncommutative effects of phase space in the time-dependent Aharonov-Bohm effect, Ann. Phys. (Amsterdam) 383, 120 (2017).

[51] K. Ma, Constrains of charge-to-mass ratios on noncommutative phase space, Adv. High Energy Phys. 2017, 1945156 (2017).

[52] S. Meljanac, D. Meljanac, F. Mercati, and D. Pikuti, Noncommutative spaces and Poincaré symmetry, Phys. Lett. B 766, 181 (2017).

[53] C.P. Martin, Computing the $\theta$-exact Seiberg-Witten map for arbitrary gauge groups, Phys. Rev. D 86, 065010 (2012).

[54] D. Brace, B. L. Cerchiai, A. F. Pasqua, U. Varadarajan, and B. Zumino, A Cohomological approach to the nonAbelian Seiberg-Witten map, J. High Energy Phys. 06 (2001) 047.

[55] G. Barnich, M. A. Grigoriev, and M. Henneaux, SeibergWitten maps from the point of view of consistent deformations of gauge theories, J. High Energy Phys. 10 (2001) 004.

[56] M. Picariello, A. Quadri, and S. P. Sorella, Chern-Simons in the Seiberg-Witten map for noncommutative Abelian gauge theories in 4-D, J. High Energy Phys. 01 (2002) 045.

[57] R. Banerjee and S. Ghosh, Seiberg-Witten map and the axial anomaly in noncommutative field theory, Phys. Lett. B 533, 162 (2002).

[58] W. Wang, J.-H Huang, and Z.-M. Sheng, Bound on noncommutative standard model with hybrid gauge transformation via lepton flavor conserving $Z$ decay, Phys. Rev. D 88, 025031 (2013).

[59] W. Wang, J.-H. Huang, and Z.-M. Sheng, TeV scale phenomenology of $e^{+} e^{-} \rightarrow \mu^{+} \mu^{-}$scattering in the noncommutative standard model with hybrid gauge transformation, Phys. Rev. D 86, 025003 (2012).

[60] W. Wang, F. Tian, and Z.-M. Sheng, Higgsstrahlung and pair production in $e^{+} e^{-}$collision in the noncommutative standard model, Phys. Rev. D 84, 045012 (2011).

[61] L. L. Foldy and S. A. Wouthuysen, On the Dirac theory of spin 1/2 particle and its nonrelativistic limit, Phys. Rev. 78, 29 (1950).

[62] O. Bertolami, J. G. Rosa, C. M. L. de Aragao, P. Castorina, and D. Zappala, Noncommutative gravitational quantum well, Phys. Rev. D 72, 025010 (2005). 